\title{
Dynamic profiling of amino acid transport and metabolism in Chinese hamster ovary cell culture
}

\author{
Sarantos Kyriakopoulos ${ }^{1}$, Karen M Polizzi ${ }^{2,3}$, Cleo Kontoravdi $i^{*}$ \\ From 23rd European Society for Animal Cell Technology (ESACT) Meeting: Better Cells for Better Health \\ Lille, France. 23-26 June 2013
}

\section{Introduction}

Chinese Hamster Ovary (CHO) cells are the most widely used industrial hosts for the production of recombinant DNA technology drugs [1]. In such processes amino acids (a.a.) are vital nutrients for growth, but also building blocks of the recombinant protein (rprotein). Our research aims to establish a better understanding of a.a. transport in and out of cells, since this could have significant impact on increasing productivity and designing feeding strategies during bioprocessing.

There are about 46 a.a. transporter proteins in mammalian cells, the genes of which are presented in Table 1 along with their substrates and all are members of the Solute Carriers (SLC) database [2]. A.a. transporters are subject to different expression profiles among mammalian cells and are grouped into more than 18 systems, based on sequence homology and function.

To our knowledge, there is no comprehensive study of a.a. transporters in industrially relevant $\mathrm{CHO}$ cells in the literature. To that direction, a.a. transporter genes were profiled during batch culture of three $\mathrm{CHO}$ cell lines with varying levels of productivity. In parallel, the intraand extracellular levels of a.a. were quantified.

\section{Materials and methods}

Three cell lines were kindly donated by Lonza Biologics. GSn8 cell line was transfected with an empty glutamine synthetase (GS) vector. GS35 and GS46 cell lines were both transfected with a GS vector that also carries the heavy and light chains of a chimeric IgG4 antibody. The specific productivity of cell line GS46, quantified by a commercial ELISA kit (Bethyl laboratories, US), is approximately double that of GS35 one.

\footnotetext{
* Correspondence: cleo.kontoravdi98@imperial.ac.uk

'Centre for Process Systems Engineering, Department of Chemical Engineering and Chemical Technology, Imperial College London, UK Full list of author information is available at the end of the article
}

Batch cultures were performed in triplicate in 1L Erlenmeyer flasks with a working volume of $300 \mathrm{~mL}$ in CD$\mathrm{CHO}$ medium (Invitrogen, UK) supplemented with $25 \mu \mathrm{M}$ MSX (Sigma, UK). Viable cell concentration was determined daily using the trypan blue dye exclusion method.

40 a.a. transporters were studied in all cell lines using real time quantitative reverse transcription polymerase chain reaction on samples from different phases of batch culture. Samples were collected at day 4 (exponential phase) and day $6 \&$ day 7 (stationary phase) of the growth curve for all cell lines (samples were also taken at day 3 for IgG4 producers only and day 9 for the null cell line only). Results are reported against the housekeeping gene "actb". Housekeeping genes "vezt" and "hirip3" were also well correlated.

The extracellular and intracellular a.a. profiles were monitored daily using high performance liquid chromatography (PicoTag, Waters, UK). Intracellular samples were quenched with $0.9 \% \mathrm{w} / \mathrm{v} \mathrm{NaCl}$ and extracted with a $50 \%$ aqueous acetonitrile solution, as described in [3].

\section{Results}

The results (Table 1) reveal that $\sim 30 \%$ of transporters are lowly expressed (fractional copies per cell), $9 \%$ are below levels of detection, whereas $40 \%$ are significantly differentially expressed either during batch cell culture, or between cell lines, or both. The remaining transporters appear to remain stable.

\section{Regulation within culture}

The majority of the transporters are found to be upregulated at stationary phase for all cell lines, as also presented in Figure 1, where a mapping of a.a. metabolism and transport has been illustrated for the null cell line. Specifically, five genes encoding for transporters of a.a. relating to the glutathione (GSH) pathway were found to be upregulated significantly higher than 2 fold at stationary phase, when 
Table 1 Amino acid transporter genes based on the SLC database [2].

\begin{tabular}{|c|c|c|c|c|c|c|c|}
\hline System & GENES & Substrates & $\begin{array}{l}\text { Expresion/ Type } \\
\text { of regulation }\end{array}$ & System & GENES & Substrates & $\begin{array}{l}\text { Expresion/ Type } \\
\text { of regulation }\end{array}$ \\
\hline \multirow[t]{3}{*}{ A } & SLC38a1 & $\begin{array}{l}\text { Ala, Asn, Cys, Gln, His, } \\
\text { Ser }\end{array}$ & $\begin{array}{l}\text { below detection } \\
\text { limits }\end{array}$ & PAT & SLC36a1 & $\begin{array}{l}\text { Gly, Ala, Pro, } \beta \text { - Ala, } \\
\text { Tau }\end{array}$ & remains stable \\
\hline & SLC38a2 & $\begin{array}{l}\text { Ala, Asn, Cys, Gln, Gly, } \\
\text { His, Met, Pro, Ser }\end{array}$ & between cell lines ${ }^{b}$ & & SLC36a2 & Gly, Ala, Pro & low $w^{a}$ \\
\hline & SLC38a4 & $\begin{array}{l}\text { Ala, Asn, Cys, Gly, Ser, } \\
\text { Thr }\end{array}$ & within cell culture ${ }^{c}$ & & SLC36a3 & putative & low ${ }^{a}$ \\
\hline \multirow[t]{2}{*}{ ASC } & SLC1a4 & Ala, Ser, Cys, Thr & within cell culture ${ }^{c}$ & & SLC36a4 & Ala, Pro, Trp & remains stable \\
\hline & SLC1a5 & $\begin{array}{l}\text { Ala, Ser, Cys, Thr, Gln, } \\
\text { Asn }\end{array}$ & both $^{d}$ & $T$ & SLC16a10 & Phe, Tyr, Trp & low ${ }^{a}$ \\
\hline asc & $\frac{\text { SLC7a10/ }}{\text { SLC3a2 }}$ & Ala, Cys, Gly, Ser, Thr & $\operatorname{low}^{a}$ & $\mathrm{X}_{\mathrm{AG}}^{-}$ & SLC1a1 & Asp, Glu & $\operatorname{low}^{a}$ \\
\hline \multirow[t]{2}{*}{$\mathrm{B}^{0}$} & SLC6a19 & Pro, Leu, Val, Ile, Met & $\operatorname{low}^{\mathrm{a}}$ & & SLC1a2 & Asp, Glu & both $^{d}$ \\
\hline & SLC6a15 & Pro, Leu, Val, Ile, Met & remains stable & & SLC1a3 & Asp, Glu & between cell lines ${ }^{b}$ \\
\hline$\overline{\mathrm{B}^{0,+}}$ & SLC6a14 & basic \& neutral a.a. & not checked & & SLC1a6 & Asp, Glu & $\begin{array}{l}\text { below detection } \\
\text { limits }\end{array}$ \\
\hline$\overline{b^{0,+}}$ & $\frac{\text { SLC7a9/ }}{\text { SLC3a1 }}$ & Arg, Lys, Cystine & $\operatorname{low}^{a}$ & & SLC1a7 & Asp, Glu & $\begin{array}{l}\text { below detection } \\
\text { limits }\end{array}$ \\
\hline$\beta$ & SLC6a6 & Tau, $\beta$-Ala & both $^{d}$ & $\overline{x_{c}}$ & $\frac{\text { SLC7a11/ }}{\text { SLC3a2 }}$ & Glu, Cystine & within cell culture ${ }^{c}$ \\
\hline \multirow[t]{3}{*}{ Gly } & SLC6a9 & Gly & within cell culture ${ }^{c}$ & $y^{+}$ & SLC7a1 & Arg, Lys, His & both $^{d}$ \\
\hline & SLC6a5 & Gly & low ${ }^{a}$ & & SLC7a2 & Arg, Lys, His & $\operatorname{low}^{\mathrm{a}}$ \\
\hline & SLC6a18 & Gly & $\begin{array}{l}\text { below detection } \\
\text { limits }\end{array}$ & & SLC7a3 & Arg, Lys, His & $\operatorname{low}^{\mathrm{a}}$ \\
\hline IMINO & SLC6a20 & Pro & $\operatorname{low}^{\mathrm{a}}$ & $y^{+} L$ & $\frac{\mathrm{SLC7a7/}}{\mathrm{SLC} 3 \mathrm{a} 2}$ & $\begin{array}{l}\text { Lys, Arg, Gln, His, Leu, } \\
\text { Met }\end{array}$ & both $^{d}$ \\
\hline \multirow[t]{5}{*}{$\mathrm{L}$} & $\frac{\mathrm{SLC7a} 5 /}{\mathrm{SLC} 3 \mathrm{a} 2}$ & $\begin{array}{l}\text { Cys, Leu, Phe, Trp, Val, } \\
\text { Tyr, lle, His, Met }\end{array}$ & both $^{d}$ & & $\frac{\mathrm{SLC7ab/}}{\mathrm{SLC} 3 \mathrm{a} 2}$ & $\begin{array}{l}\text { Lys, Arg, Gln, His, Leu, } \\
\text { Met, Ala, Cys }\end{array}$ & remains stable \\
\hline & $\frac{\mathrm{SLC7a8/}}{\mathrm{SLC} 3 \mathrm{a} 2}$ & neutral a.a., except Pro & $\operatorname{low}^{a}$ & $\begin{array}{l}\text { His \& small } \\
\text { peptides }\end{array}$ & SLC15a3 & His & between cell lines ${ }^{b}$ \\
\hline & SLC43a1 & Leu, lle, Met, Phe & low ${ }^{a}$ & & SLC15a4 & His & between cell lines ${ }^{b}$ \\
\hline & SLC43a2 & Leu, lle, Met, Phe & between cell lines ${ }^{b}$ & $\begin{array}{l}\text { Heavy subunits of } \\
\text { hetero-meric }\end{array}$ & SLC3a1 & $\begin{array}{l}\text { various based on } \\
\text { "partner" }\end{array}$ & low \\
\hline & SLC43a3 & putative & between cell lines ${ }^{b}$ & & SLC3a2 & $\begin{array}{l}\text { various based on } \\
\text { "partner" }\end{array}$ & both $^{d}$ \\
\hline \multirow[t]{4}{*}{$\mathrm{N}$} & SLC38a3 & Ala, Asn, Gln, His & not checked & Not in a system & SLC6a7 & Pro & not checked \\
\hline & SLC38a5 & Gln, Asn, His, Ser & both $^{d}$ & & SLC6a17 & neutral a.a. & not checked \\
\hline & & & & & SLC7a13 & Asp, Glu & not checked \\
\hline & & & & & SLC12A8 & putative & not checked \\
\hline
\end{tabular}

The "Expression/ Type of regulation" column refers to our results for the CHO cell lines described in the materials \& methods section: ${ }^{2}$ low levels-refers to fractional copies per cell; ${ }^{b}$ regulation between cell lines-refers to regulation significantly higher than two fold at least at a time point between the different cell lines presented; ' regulation within cell culture-refers to differential expression (significantly higher than two fold) at least at a time point within cell culture of a given cell line; ${ }^{d}$ both types of regulation-refers to a gene presenting both ${ }^{b}$ and ${ }^{c}$ as discussed previously.

compared to exponential phase for all cell lines. These genes were: slc1a4 (Ala and Cys), slc6a9 (Gly), slc1a2 (Glu and Asp), slc7a11 (Cystine and Glu), and heteromeric transporter slc3a2 which partners with slc7a11. GSH is a well-known marker of oxidative stress [4], high levels of which have been associated with high productivity [5].

\section{Regulation between cell lines}

In their majority, genes were found to be upregulated for protein producing cell lines at all time points. Genes whose expression is upregulated significantly (two-fold or higher) in the protein-producers at all time points analyzed were: slc43a2 (system L, leucine and branchedchain a.a.) and slc1a2 (system $\mathrm{X}_{\mathrm{AG}}^{-}$, glutamate and aspartate). However, no genes, apart from slc6a6 (taurine and b-Ala), were found to be differentially expressed between high (GS46) and low producer (GS35). We find slc6a6 gene differentially expressed early in cell culture (day 3), which makes us hypothesize that the gene could be a candidate for selection purposes. The overexpression of 


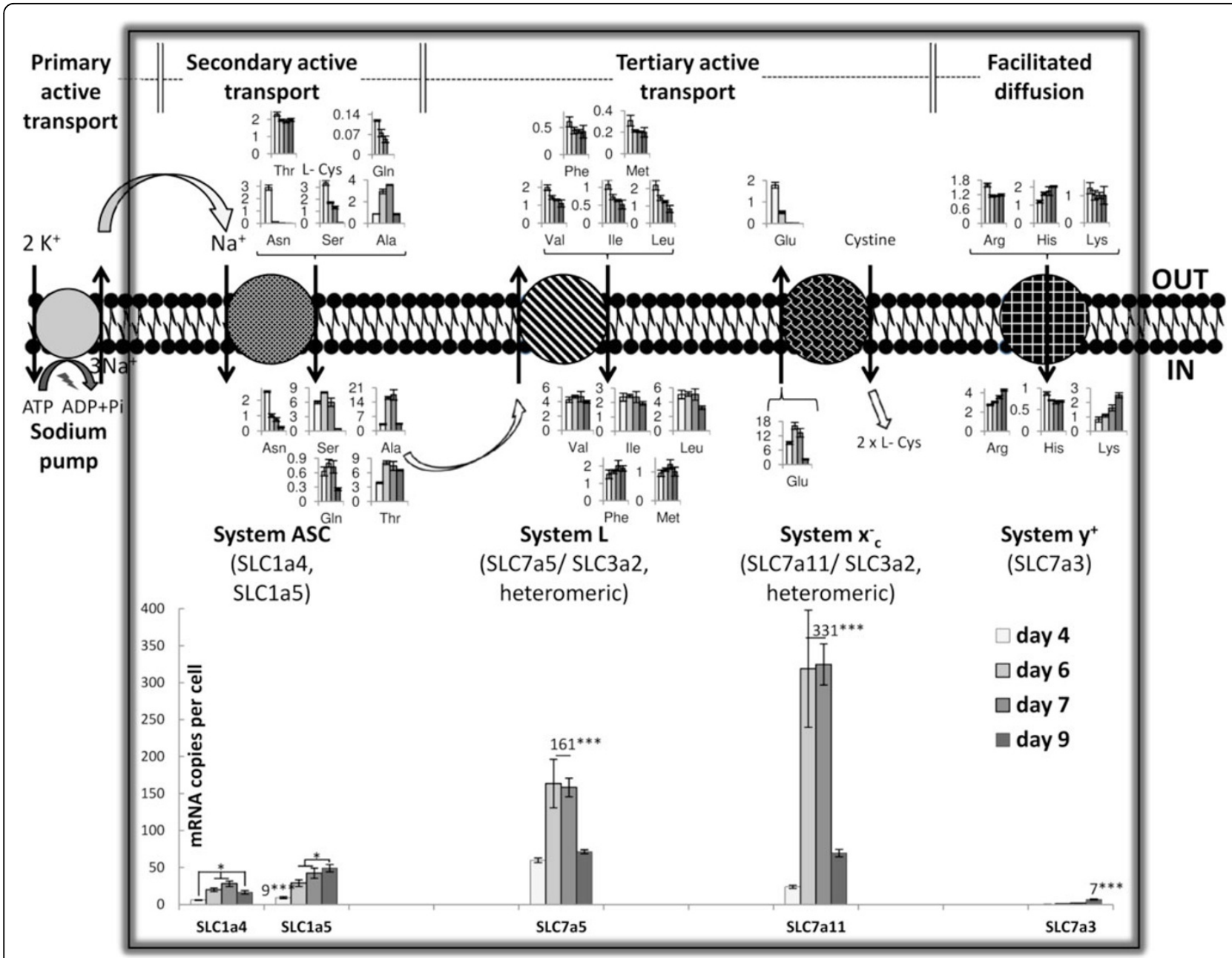

Figure $1 \mathrm{~A}$ map associating the differentially expressed amino acid transporters for the null cell line, their amino acid substrates, and the intracellular concentrations (femtomol/ cell, in the area designated by the "IN" tag) and extracellular concentrations (mM, in the area designated by the "OUT" tag) of the latter. A.a. transport is highlighted by the black box. The expression of the mRNA levels of the differentially expressed a.a. transporters (in mRNA copies per cell) at different phases of cell culture, exponential (day 4), stationary (days 6 \& 7), and decline (day 9) is displayed at the bottom, where stationary phase samples are averaged, since not statistically different (for ease of statistical analysis visualization). The relevant energy utilisation mechanisms of each system are also depicted (top). Genes: slc6a9 (glycine), slc1a2 (acidic a. a.), slc7a7 (basic and branched chain a.a.) and its heteromeric transporter slc3a2 were also found to be differentially expressed, but are not presented in this figure. Our chosen a.a. analysis method was not able to quantify cysteine (L-Cys) levels.

this gene in $\mathrm{CHO}$ cells has been found to significantly enhance growth and productivity [6].

\section{Feeding strategy based on order of feeding}

The a.a. transporters gene expression findings correlate well with the extracellular and intracellular concentration profiles of their respective substrates (Figure 1). By analysing the differentially expressed genes for a specific cell line a feeding strategy can be designed. For example, we find transporter slc7a5, of system L, highly upregulated at stationary phase for the null cell line (Figure 1). This transporter exchanges an intracellular neutral a.a. with an extracellular branched chain one (isoleucine, leucine, valine). Branched chain amino acids are associated with the mTor signalling pathway, essential regulator for many physiological roles in mammalian cells [7]. Hence, a feeding strategy can be proposed, where neutral amino acids are fed first and followed by branched chain amino acids, in order for them to be more effectively uptaken. A similar type of pre-conditioning was found to significantly enhance cellular protein production in another type of mammalian cells [7].

\section{Conclusions}

Glutathione pathway associated a.a. transporters (slc1a2, slc1a4, slc6a9, slc7a11/ slc3a2) can be targeted as genetic engineering targets, since are all found highly upregulated at stationary phase of cell culture. Additionally, 
transporters slc1a2, slc43a2 are associated with rprotein productivity, since all of them are found to be upregulated for producing cell lines vs the null. Gene slc6a6, carrying taurine and $\beta$-alanine, can be associated with high productivity (as also suggested in [6]), as was also found to be differentially expressed in the high vs the low producer early in cell culture. A feeding strategy can be proposed, based on our results that remains to be tested experimentally. Finally, extending this integrative approach to the proteome level would help link regulation at the transcriptomic level to actual differences in transport capability.

\section{Acknowledgements}

S.K. would like to thank EPSRC \& iChemE for financial support. K.P. would like to thank RCUK and C.K. thanks RCUK \& Lonza Biologics for their Fellowships.

\section{Authors' details}

${ }^{1}$ Centre for Process Systems Engineering, Department of Chemical Engineering and Chemical Technology, Imperial College London, UK. ${ }^{2}$ Division of Molecular Biosciences, Imperial College London, UK. ${ }^{3}$ Centre for Synthetic Biology and Innovation, Imperial College London, UK.

Published: 4 December 2013

\section{References}

1. Kyriakopoulos S, Kontoravdi C: Analysis of the landscape of biologicallyderived pharmaceuticals in Europe: Dominant production systems, molecule types on the rise and approval trends. European journal of pharmaceutical sciences: official journal of the European Federation for Pharmaceutical Sciences 2012, 48:428-441.

2. Hediger MA, Romero MF, Peng JB, Rolfs A, Takanaga $H$, Bruford EA: The $A B C s$ of solute carriers: physiological, pathological and therapeutic implications of human membrane transport proteins - Introduction. Pflug Arch Eur J Phy 2004, 447:465-468.

3. Dietmair S, Timmins NE, Gray PP, Nielsen LK, Kromer JO: Towards quantitative metabolomics of mammalian cells: Development of a metabolite extraction protocol. Anal Biochem 2010, 404:155-164

4. Selvarasu S, Ho YS, Chong WPK, Wong NSC, Yusufi FNK, Lee YY, Yap MGS, Lee DY: Combined in silico modeling and metabolomics analysis to characterize fed-batch CHO cell culture. Biotechnol Bioeng 2012, 109:1415-1429.

5. Chong WP, Thng SH, Hiu AP, Lee DY, Chan EC, Ho YS: LC-MS-based metabolic characterization of high monoclonal antibody-producing Chinese hamster ovary cells. Biotechnol Bioeng 2012, 109:3103-3111.

6. Tabuchi H, Sugiyama T, Tanaka S, Tainaka S: Overexpression of Taurine Transporter in Chinese Hamster Ovary Cells Can Enhance Cell Viability and Product Yield, While Promoting Glutamine Consumption. Biotechnol Bioeng 2010, 107:998-1003.

7. Nicklin P, Bergman P, Zhang BL, Triantafellow E, Wang H, Nyfeler B, Yang HD, Hild M, Kung C, Wilson C, et al: Bidirectional Transport of Amino Acids Regulates mTOR and Autophagy. Cell 2009, 136:521-534.

doi:10.1186/1753-6561-7-S6-P97

Cite this article as: Kyriakopoulos et al: Dynamic profiling of amino acid transport and metabolism in Chinese hamster ovary cell culture. BMC Proceedings 2013 7(Suppl 6):P97.

\section{Submit your next manuscript to BioMed Central and take full advantage of:}

- Convenient online submission

- Thorough peer review

- No space constraints or color figure charges

- Immediate publication on acceptance

- Inclusion in PubMed, CAS, Scopus and Google Scholar

- Research which is freely available for redistribution 